\title{
WEIGHTED SELBERG ORTHOGONALITY AND UNIQUENESS OF FACTORIZATION OF AUTOMORPHIC $L$-FUNCTIONS
}

\author{
JIANYA LIU ${ }^{12}$ AND YANGBO YE ${ }^{32}$
}

\begin{abstract}
We prove a weighted version of Selberg's orthogonality conjecture for automorphic $L$-functions attached to irreducible cuspidal representations of $G L_{m}$ over $\mathbb{Q}$. Using this weighted orthogonality, we obtain the uniqueness of factorization of general $L$-functions. As a consequence, we prove that the $L$-function attached to an automorphic irreducible cuspidal representation of $G L_{m}$ over $\mathbb{Q}$ cannot be factored further.
\end{abstract}

1. Introduction. It is a far-reaching conjecture of Langlands [11] that the most general $L$-function is indeed the $L$-function $L(s, \Pi)$ attached to an automorphic representation $\Pi$ of $G L_{n}$ over an algebraic number field. It was further conjectured that this $L(s, \Pi)$ is equal to a product of $L$-functions $L\left(s, \pi_{j}\right)$ attached to automorphic irreducible cuspidal representations $\pi_{j}$ of $G L_{m_{j}}$ over $\mathbb{Q}$ in a unique way:

$$
L(s, \Pi)=L\left(s, \pi_{1}\right) \cdots L\left(s, \pi_{k}\right) .
$$

These $L\left(s, \pi_{j}\right)$ are called principal or primitive $L$-functions over $\mathbb{Q}$ in the sense that they are supposed to be $L$-functions that cannot be factored further. They are believed to be the building blocks of all $L$-functions.

A known special case of the unique factorization (1.1) is for $\Pi$ being an automorphic irreducible cuspidal representation of $G L_{n}$ over a cyclic algebraic number field $F$, when $\Pi$ is invariant under the action of the $\operatorname{Gal}(F / \mathbb{Q})$. According to Arthur and Clozel [1], such a representation $\Pi$ is the base change of $\pi \otimes \eta$, where $\pi$ is an automorphic irreducible cuspidal representation of $G L_{n}$ over $\mathbb{Q}$, and $\eta$ is any idele class character of $\mathbb{Q}$ trivial on $\mathrm{N}_{F / \mathbb{Q}}\left(F_{\mathbb{A}}^{\times}\right)$. In terms of $L$-functions, we have the factorization

$$
L(s, \Pi)=\prod_{\eta} L(s, \pi \otimes \eta)
$$

2000 Mathematics Subject Classification (MSC) 11F70, 11M26, $11 \mathrm{M} 41$.

${ }^{1}$ Partially supported by China NNSF Grant Number 10125101

${ }^{2}$ Partially supported by a China NNSF Dual Research Base Grant

${ }^{3}$ Project sponsored by the USA NSA under Grant Number MDA904-03-1-0066.

The United States Government is authorized to reproduce and distribute reprints notwithstanding any copyright notation herein. 
uniquely.

In this paper we will prove the uniqueness of the factorization in (1.1). That is, if any general $L$-function can be written as a product of principal $L$-functions $L\left(s, \pi_{j}\right)$ for $G L_{m_{j}}$ over $\mathbb{Q}$, we will show that this factorization is unique.

ThEOREM 1.1. Let $\pi_{j}$ and $\pi_{i}^{\prime}, j=1, \ldots, k, i=1, \ldots, l$, be automorphic irreducible cuspidal representations of $G L_{m_{j}}\left(\mathbb{Q}_{\mathbb{A}}\right)$ and $G L_{m_{i}^{\prime}}\left(\mathbb{Q}_{\mathbb{A}}\right)$ with unitary central characters, respectively. Then

$$
L\left(s, \pi_{1}\right) \cdots L\left(s, \pi_{k}\right)=L\left(s, \pi_{1}^{\prime}\right) \cdots L\left(s, \pi_{l}^{\prime}\right)
$$

cannot hold, if there is a $\pi_{j}$ which is not equivalent to any $\pi_{i}^{\prime}$.

By taking $k=1$, Theorem 1.1 implies that $L\left(s, \pi_{1}\right)$ cannot be factored further.

COROLlary. The $L$-function $L(s, \pi)$ attached to an automorphic irreducible cuspidal representation $\pi$ of $G L_{m}\left(\mathbb{Q}_{\mathbb{A}}\right)$ cannot be factored into $L\left(s, \pi_{1}^{\prime}\right) \cdots L\left(s, \pi_{l}^{\prime}\right)$ nontrivially, where $\pi_{i}^{\prime}$ is an automorphic irreducible cuspidal representation of $G L_{m_{i}^{\prime}}\left(\mathbb{Q}_{\mathbb{A}}\right)$ with unitary central character.

Unique factorization of $L$-functions in the Selberg class (Selberg [18]) has been studied by Conrey and Ghosh [2] and Ram Murty [14], under the Selberg orthogonality conjecture (Conjecture 1.1 below). For automorphic $L$-functions, Ram Murty [15] proved that $L(s, \pi)$ is primitive, i.e., cannot be factored further, when $\pi$ is an automorphic irreducible cuspidal representation of $G L_{2}\left(\mathbb{Q}_{\mathbb{A}}\right)$, under the Ramanujan conjecture. Our proof of Theorem 1.1 and its Corollary is unconditional.

The proof of Theorem 1.1 will utilize a weighted version of Selberg's orthogonality for automorphic $L$-functions. Let $\pi$ be an automorphic irreducible cuspidal representation of $G L_{m}\left(\mathbb{Q}_{\mathbb{A}}\right)$. Then the global $L$-functions attached to $\pi$ are given by products of local factors for Re $s>1$ (Godement and Jacquet [5]):

$$
\begin{aligned}
& L(s, \pi)=\prod_{p} L_{p}\left(s, \pi_{p}\right), \\
& \Phi(s, \pi)=L_{\infty}\left(s, \pi_{\infty}\right) L(s, \pi),
\end{aligned}
$$

where

$$
L_{p}\left(s, \pi_{p}\right)=\prod_{j=1}^{m}\left(1-\alpha_{\pi}(p, j) p^{-s}\right)^{-1}
$$


and

$$
L_{\infty}\left(s, \pi_{\infty}\right)=\prod_{j=1}^{m} \Gamma_{\mathbb{R}}\left(s+\mu_{\pi}(j)\right) .
$$

Here $\Gamma_{\mathbb{R}}(s)=\pi^{-s / 2} \Gamma(s / 2)$, and $\alpha_{\pi}(p, j)$ and $\mu_{\pi}(j), j=1, \ldots, m$, are complex numbers associated with $\pi_{p}$ and $\pi_{\infty}$, respectively, according to the Langlands correspondence. Denote

$$
a_{\pi}\left(p^{k}\right)=\sum_{1 \leq j \leq m} \alpha_{\pi}(p, j)^{k}
$$

Then for $\operatorname{Re} s>1$, we have

$$
\frac{d}{d s} \log L(s, \pi)=-\sum_{n \geq 1} \frac{\Lambda(n) a_{\pi}(n)}{n^{s}}
$$

where $\Lambda(n)=\log p$ if $n=p^{k}$ and $=0$ otherwise. If $\pi^{\prime}$ is an automorphic irreducible cuspidal representation of $G L_{m^{\prime}}\left(\mathbb{Q}_{\mathbb{A}}\right)$, we define $L\left(s, \pi^{\prime}\right), \alpha_{\pi^{\prime}}(p, i), \mu_{\pi^{\prime}}(i)$, and $a_{\pi^{\prime}}\left(p^{k}\right)$ likewise, for $i=1, \ldots, m^{\prime}$. If $\pi$ and $\pi^{\prime}$ are equivalent, then $m=m^{\prime}$ and $\left\{\alpha_{\pi}(p, j)\right\}=$ $\left\{\alpha_{\pi^{\prime}}(p, i)\right\}$ for any $p$. Hence $a_{\pi}(n)=a_{\pi^{\prime}}(n)$ for any $n=p^{k}$, when $\pi \cong \pi^{\prime}$.

The Selberg orthogonality conjecture for automorphic $L$-functions $L(s, \pi)$ was proposed in 1989 (Selberg [18]). See also Ram Murty [14].

CONJECTURe 1.1. (i) For any automorphic irreducible cuspidal representation $\pi$ of $G L_{m}\left(\mathbb{Q}_{\mathbb{A}}\right)$

$$
\sum_{p \leq x} \frac{\left|a_{\pi}(p)\right|^{2}}{p}=\log \log x+O(1) .
$$

(ii) For any automorphic irreducible cuspidal representations $\pi$ and $\pi^{\prime}$ of $G L_{m}\left(\mathbb{Q}_{\mathbb{A}}\right)$ and $G L_{m^{\prime}}\left(\mathbb{Q}_{\mathbb{A}}\right)$, respectively,

$$
\sum_{p \leq x} \frac{a_{\pi}(p) \bar{a}_{\pi^{\prime}}(p)}{p} \ll 1
$$

if $\pi$ is not equivalent to $\pi^{\prime}$.

A weaker version of this conjecture is

Conjecture 1.2. (i) For any automorphic irreducible cuspidal representation $\pi$ of $G L_{m}\left(\mathbb{Q}_{\mathbb{A}}\right)$

$$
\sum_{n \leq x} \frac{(\log n) \Lambda(n)\left|a_{\pi}(n)\right|^{2}}{n}=\frac{1}{2} \log ^{2} x+O(\log x) .
$$


(ii) For any automorphic irreducible cuspidal representations $\pi$ and $\pi^{\prime}$ of $G L_{m}\left(\mathbb{Q}_{\mathbb{A}}\right)$ and $G L_{m^{\prime}}\left(\mathbb{Q}_{\mathbb{A}}\right)$, respectively,

$$
\sum_{n \leq x} \frac{(\log n) \Lambda(n) a_{\pi}(n) \bar{a}_{\pi^{\prime}}(n)}{n} \ll \log x,
$$

if $\pi$ is not equivalent to $\pi^{\prime}$.

Note that in (1.6) and (1.7), the sums are taken over primes $(n=p)$ and prime powers $\left(n=p^{k}, k>1\right)$, while in Conjecture 1.1, the sums are taken over primes only. Rudnick and Sarnak conjectured that the sums taken over prime powers contained in (1.6) and (1.7) converge as $x \rightarrow \infty$ :

Conjecture 1.3. ([17]) For any fixed $k \geq 2$,

$$
\sum_{p} \frac{\left|(\log p) a_{\pi}\left(p^{k}\right)\right|^{2}}{p^{k}}<\infty
$$

Conjectures 1.2 and 1.3 imply Conjecture 1.1.

Conjecture 1.2 Part (i) in (1.6) was proved by Rudnick and Sarnak [17]. They also proved Conjecture 1.3 for $G L_{2}$ and $G L_{3}$. Recently, Kim and Sarnak [10] proved Conjecture 1.3 for $G L_{4}$. Therefore, part (i) of Selberg's original Conjecture 1.1 is known up to $G L_{4}$. On the other hand, part (ii) of Conjectures 1.1 and 1.2 is still open.

In [17], Rudnick and Sarnak first proved a weighted version of (1.6):

$$
\sum_{n \leq x}\left(1-\frac{n}{x}\right) \frac{(\log n) \Lambda(n)\left|a_{\pi}(n)\right|^{2}}{n}=\frac{1}{2} \log ^{2} x+O(\log x)
$$

and deduced (1.6) from (1.8) using the fact that the left side of (1.6) is a sum of positive terms. What we want to prove here is a weighted version of (1.7).

THEOREM 1.2. For any automorphic irreducible cuspidal representations $\pi$ and $\pi^{\prime}$ of $G L_{m}\left(\mathbb{Q}_{\mathbb{A}}\right)$ and $G L_{m^{\prime}}\left(\mathbb{Q}_{\mathbb{A}}\right)$, respectively,

$$
\sum_{n \leq x}\left(1-\frac{n}{x}\right) \frac{(\log n) \Lambda(n) a_{\pi}(n) \bar{a}_{\pi^{\prime}}(n)}{n} \ll \log x,
$$

if $\pi$ is not equivalent to $\pi^{\prime}$.

We note that we cannot remove the weight $1-n / x$ from the left side of (1.9) using standard methods, because the sum there is not of positive terms. For our purpose of proving Theorem 1.1, however, there is no need to remove this weight. There is also no need to remove terms on prime powers from the sum on the left side of (1.9). That is, we do not assume Conjecture 1.3. 
When $\pi$ and $\pi^{\prime}$ are not twisted equivalent, i.e., when $\pi \neq \pi^{\prime} \otimes \alpha^{i t}$ for any $t \in \mathbb{R}$, where $\alpha(g)=|\operatorname{det}(g)|$, (1.9) was proved by the authors in [12]. In this paper, we will prove the remaining case: (1.9) is true when $m=m^{\prime}$ and $\pi \cong \pi^{\prime} \otimes \alpha^{i \tau_{0}}$ for some non-zero $\tau_{0} \in \mathbb{R}$.

We would like to express our sincere thanks to Peter Sarnak and Freydoon Shahidi for helpful information and discussion.

2. Proof of Theorem 1.1. We first prove Theorem 1.1 using (1.8) and Theorem 1.2. A similar argument was first used by Conrey and Ghosh [2] who deduced the uniqueness of factorization in the Selberg class by assuming Conjecture 1.1 (cf. Ram Murty [14]).

Suppose that (1.2) holds. Taking logarithmic derivatives of both sides like in (1.3), we get

$$
-\sum_{n \geq 1} \frac{\Lambda(n)}{n^{s}}\left(a_{\pi_{1}}(n)+\cdots+a_{\pi_{k}}(n)\right)=-\sum_{n \geq 1} \frac{\Lambda(n)}{n^{s}}\left(a_{\pi_{1}^{\prime}}(n)+\cdots+a_{\pi_{l}^{\prime}}(n)\right)
$$

for $\operatorname{Re} s>1$. Consequently

$$
a_{\pi_{1}}(n)+\cdots+a_{\pi_{k}}(n)=a_{\pi_{1}^{\prime}}(n)+\cdots+a_{\pi_{l}^{\prime}}(n)
$$

for any $n=p^{k} \geq 1$. Consider the sum

$$
\begin{aligned}
& \sum_{n \leq x}\left(1-\frac{n}{x}\right) \frac{\Lambda(n) \log n}{n}\left(a_{\pi_{1}}(n)+\cdots+a_{\pi_{k}}(n)\right) \bar{a}_{\pi_{j}}(n) \\
& \quad=\sum_{n \leq x}\left(1-\frac{n}{x}\right) \frac{\Lambda(n) \log n}{n}\left(a_{\pi_{1}^{\prime}}(n)+\cdots+a_{\pi_{l}^{\prime}}(n)\right) \bar{a}_{\pi_{j}}(n) .
\end{aligned}
$$

Since $\pi_{j}$ is not equivalent to any $\pi_{i}^{\prime}$, the right side of $(2.1)$ is $\ll \log x$, by Theorem 1.2. On the left side of (2.1), we get at least one, and possibly more copies of $\frac{1}{2} \log ^{2} x+O(\log x)$ by (1.8). This contradiction proves Theorem 1.1.

3. Rankin-Selberg $L$-functions. We will use the Rankin-Selberg $L$-functions $L\left(s, \pi \times \pi^{\prime}\right)$ as developed by Jacquet, Piatetski-Shapiro, and Shalika [6], Shahidi [19], and Moeglin and Waldspurger [13], where $\pi$ and $\pi^{\prime}$ are automorphic irreducible cuspidal representations of $G L_{m}$ and $G L_{m^{\prime}}$, respectively, over $\mathbb{Q}$ with unitary central characters. This $L$-function is also given by local factors:

$$
L\left(s, \pi \times \pi^{\prime}\right)=\prod_{p} L_{p}\left(s, \pi_{p} \times \pi_{p}^{\prime}\right)
$$

where

$$
L_{p}\left(s, \pi_{p} \times \pi_{p}^{\prime}\right)=\prod_{j=1}^{m} \prod_{k=1}^{m^{\prime}}\left(1-\alpha_{\pi}(p, j) \alpha_{\pi^{\prime}}(p, k) p^{-s}\right)^{-1}
$$


The Archimedean local factor $L_{\infty}\left(s, \pi_{\infty} \times \pi_{\infty}^{\prime}\right)$ is defined by

$$
L_{\infty}\left(s, \pi_{\infty} \times \pi_{\infty}^{\prime}\right)=\prod_{j=1}^{m} \prod_{k=1}^{m^{\prime}} \Gamma_{\mathbb{R}}\left(s+\mu_{\pi \times \pi^{\prime}}(j, k)\right)
$$

where, when $\pi_{\infty}$ and $\pi_{\infty}^{\prime}$ are unramified,

$$
\left\{\mu_{\pi \times \pi^{\prime}}(j, k): 1 \leq j \leq m, 1 \leq k \leq m^{\prime}\right\}=\left\{\mu_{\pi}(j)+\mu_{\pi^{\prime}}(k): 1 \leq j \leq m, 1 \leq k \leq m^{\prime}\right\}
$$

Denote

$$
\Phi\left(s, \pi \times \pi^{\prime}\right)=L_{\infty}\left(s, \pi_{\infty} \times \pi_{\infty}^{\prime}\right) L\left(s, \pi \times \pi^{\prime}\right) .
$$

We will need the following properties of the $L$-functions $L\left(s, \pi \times \pi^{\prime}\right)$ and $\Phi\left(s, \pi \times \pi^{\prime}\right)$.

RS1. The Euler product for $L\left(s, \pi \times \pi^{\prime}\right)$ in (3.1) converges absolutely for Re $s>1$ (Jacquet and Shalika [7] ).

RS2. The complete $L$-function $\Phi\left(s, \pi \times \pi^{\prime}\right)$ has an analytic continuation to the entire complex plane and satisfies a functional equation

$$
\Phi\left(s, \pi \times \pi^{\prime}\right)=\varepsilon\left(s, \pi \times \pi^{\prime}\right) \Phi\left(1-s, \tilde{\pi} \times \tilde{\pi}^{\prime}\right),
$$

with

$$
\varepsilon\left(s, \pi \times \pi^{\prime}\right)=\tau\left(\pi \times \pi^{\prime}\right) Q_{\pi \times \pi^{\prime}}^{-s}
$$

where $Q_{\pi \times \pi^{\prime}}>0$ and $\tau\left(\pi \times \pi^{\prime}\right)= \pm Q_{\pi \times \pi^{\prime}}^{1 / 2}$ (Shahidi [19], [20], [21], and [22]).

RS3. When $\tilde{\pi} \not \pi^{\prime} \otimes \alpha^{i t}$ for any $t \in \mathbb{R}, \Phi\left(s, \pi \times \pi^{\prime}\right)$ is holomorphic. When $m=m^{\prime}$ and $\tilde{\pi} \cong \pi^{\prime} \otimes \alpha^{i \tau_{0}}$ for some $\tau_{0} \in \mathbb{R}$, the only poles of $\Phi\left(s, \pi \times \pi^{\prime}\right)$ are simple poles at $s=i \tau_{0}$ and $1+i \tau_{0}$ coming from $L\left(s, \pi \times \pi^{\prime}\right)$ (Jacquet and Shalika [7], [8], Moeglin and Waldspurger [13]). We will only consider the latter case in the proof of Theorem 1.2.

RS4. $\Phi\left(s, \pi \times \pi^{\prime}\right)$ is meromorphic of order one away from its poles, and bounded in vertical strips (Gelbart and Shahidi [4]).

RS5. $\Phi\left(s, \pi \times \pi^{\prime}\right)$ and $L\left(s, \pi \times \pi^{\prime}\right)$ are non-zero in $\operatorname{Re}(s) \geq 1$. (Shahidi [19])

In addition, we will also use the following simple properties of the $\Gamma$-function. If

$$
\lambda(s)=\min _{n \leq 0}|s-n|,
$$

then

$$
-\frac{d}{d s} \log \Gamma(s) \ll \frac{1}{\lambda(s)}+\log (|s|+2),
$$


and

$$
\frac{d^{2}}{d s^{2}} \log \Gamma(s) \ll \frac{1}{\lambda(s)}
$$

For the proof of (3.2), see e.g. Pan and Pan [16] p.49. To show (3.3), one appeals to Pan and Pan $[16]$ p.48, to get

$$
-\frac{d}{d s} \log \Gamma(s)=\frac{1}{s}+\gamma+\sum_{n=1}^{\infty}\left(\frac{1}{n+s}-\frac{1}{n}\right)
$$

where $\gamma$ is Euler's constant. Therefore

$$
\frac{d^{2}}{d s^{2}} \log \Gamma(s)=\frac{1}{s^{2}}+\sum_{n=1}^{\infty} \frac{1}{(n+s)^{2}}=\sum_{n=0}^{\infty} \frac{1}{(n+s)^{2}} \ll \frac{1}{\lambda(s)} .
$$

Let $\mathbb{C}(\delta)$ be the complex plane with the discs $|s-n|<\delta, n=0,-1,-2, \cdots$ excluded. Then by (3.2) and (3.3), for $s \in \mathbb{C}(\delta)$ we have

$$
-\frac{d}{d s} \log \Gamma(s) \ll_{\delta} \log (|s|+2), \quad \frac{d^{2}}{d s^{2}} \log \Gamma(s) \ll_{\delta} 1 .
$$

Lemma 3.1. Assume $m=m^{\prime}$ and $\tilde{\pi} \cong \pi^{\prime} \otimes \alpha^{i \tau_{0}}$ for some nonzero $\tau_{0} \in \mathbb{R}$. For any $T$

$$
\sum_{\rho} \frac{1}{1+(\operatorname{Im}(\rho)-T)^{2}} \ll \log \left(Q_{\pi \times \pi^{\prime}}(|T|+2)\right)
$$

where $\rho$ runs over all the non-trivial zeros of $L\left(s, \pi \times \pi^{\prime}\right)$.

Proof. Since $\Phi\left(s, \pi \times \pi^{\prime}\right)$ is of order one (RS4), we have (see e.g. Davenport [3] Chapter 11)

$$
\Phi\left(s, \pi \times \pi^{\prime}\right)=\frac{e^{A+B s}}{\left(s-\left(1+i \tau_{0}\right)\right)\left(s-i \tau_{0}\right)} \prod_{\rho}\left(1-\frac{s}{\rho}\right) e^{s / \rho},
$$

where $A=A_{\pi \times \pi^{\prime}}$ and $B=B_{\pi \times \pi^{\prime}}$ are constants depending on $\pi \times \pi^{\prime}$. Take logarithmic derivative

$$
\frac{d}{d s} \log \Phi\left(s, \pi \times \pi^{\prime}\right)=B-\frac{1}{s-\left(1+i \tau_{0}\right)}-\frac{1}{s-i \tau_{0}}+\sum_{\rho}\left(\frac{1}{s-\rho}+\frac{1}{\rho}\right),
$$

where here and throughout we set $\log 1=0$. By the definition of $\Phi\left(s, \pi \times \pi^{\prime}\right)$, we have

$$
\frac{d}{d s} \log \Phi\left(s, \pi \times \pi^{\prime}\right)=\frac{d}{d s} \log L_{\infty}\left(s, \pi_{\infty} \times \pi_{\infty}^{\prime}\right)+\frac{d}{d s} \log L\left(s, \pi \times \pi^{\prime}\right) .
$$


The definition of $L_{\infty}\left(s, \pi_{\infty} \times \pi_{\infty}^{\prime}\right)$ and (3.2) give

$$
\begin{aligned}
\frac{d}{d s} \log L_{\infty}\left(s, \pi_{\infty} \times \pi_{\infty}^{\prime}\right)= & \sum_{j, k} \frac{d}{d s} \log \pi^{-\left(s+\mu_{\pi \times \pi^{\prime}}(j, k)\right) / 2} \\
& +\sum_{j, k} \frac{d}{d s} \log \Gamma\left(\frac{s+\mu_{\pi \times \pi^{\prime}}(j, k)}{2}\right) \\
\ll & \sum_{j, k}\left(\lambda\left(\frac{s+\mu_{\pi \times \pi^{\prime}}(j, k)}{2}\right)^{-1}+\log (|s|+2)\right) .
\end{aligned}
$$

Let $\mathbb{C}(m)$ be the complex plane with the discs

$$
\left|s-2 n+\mu_{\pi \times \pi^{\prime}}(j, k)\right|<\frac{1}{8 m^{2}}, \quad n \leq 0, \quad 1 \leq j, k \leq m,
$$

excluded. Then for $s \in \mathbb{C}(m)$ and all $j, k$,

$$
\lambda\left(\frac{s+\mu_{\pi \times \pi^{\prime}}(j, k)}{2}\right) \geq \frac{1}{16 m^{2}} .
$$

Thus in $\mathbb{C}(m)$

$$
\frac{d}{d s} \log L_{\infty}\left(s, \pi_{\infty} \times \pi_{\infty}^{\prime}\right) \ll_{m} \log (|s|+2) .
$$

By (3.4), (3.5), and (3.6) we have

$$
\begin{aligned}
& \frac{d}{d s} \log L\left(s, \pi \times \pi^{\prime}\right) \\
& =B+\sum_{\rho}\left(\frac{1}{s-\rho}+\frac{1}{\rho}\right)-\frac{1}{s-\left(1+i \tau_{0}\right)}-\frac{1}{s-i \tau_{0}} \\
& \quad+O(\log (|s|+2)) .
\end{aligned}
$$

Here we give a remark about the structure of $\mathbb{C}(m)$. For $j, k=1, \cdots, m$, denote by $\beta(j, k)$ the fractional part of $\operatorname{Re}\left(\mu_{\pi \times \pi^{\prime}}(j, k)\right)$. (If $\pi$ and $\pi^{\prime}$ are unramified at $\mathbb{R}$, the Ramanujan conjecture predicts that $\operatorname{Re}\left(\mu_{\pi \times \pi^{\prime}}(j, k)\right)=0$. We will not assume the Ramanujan conjecture in this paper.) In addition we let $\beta(0,0)=0$ and $\beta(m+1, m+1)=1$. Then all $\beta(j, k) \in[0,1]$, and hence there exist $\beta\left(j_{1}, k_{1}\right), \beta\left(j_{2}, k_{2}\right)$ such that $\beta\left(j_{2}, k_{2}\right)-\beta\left(j_{1}, k_{1}\right) \geq$ $1 /\left(3 m^{2}\right)$ and there is no $\beta(j, k)$ lying between $\beta\left(j_{1}, k_{1}\right)$ and $\beta\left(j_{2}, k_{2}\right)$. It follows that the strip $S_{0}=\left\{s: \beta\left(j_{1}, k_{1}\right)+1 /\left(8 m^{2}\right) \leq \operatorname{Re}(s) \leq \beta\left(j_{2}, k_{2}\right)-1 /\left(8 m^{2}\right)\right\}$ is contained in $\mathbb{C}(m)$. Consequently, for all $n=0,-1,-2, \cdots$, the strips

$$
S_{n}=\left\{s: n+\beta\left(j_{1}, k_{1}\right)+1 /\left(8 m^{2}\right) \leq \operatorname{Re}(s) \leq n+\beta\left(j_{2}, k_{2}\right)-1 /\left(8 m^{2}\right)\right\}
$$

are subsets of $\mathbb{C}(m)$. This structure of $\mathbb{C}(m)$ will be used later. We will prove in a moment that

$$
\operatorname{Re}(B)=-\sum_{\rho} \operatorname{Re} \frac{1}{\rho}+O\left(\log Q_{\pi \times \pi^{\prime}}\right) .
$$


Now taking real part in (3.7), we get by (3.9) that

$$
\begin{aligned}
\operatorname{Re} \frac{d}{d s} & \log L\left(s, \pi \times \pi^{\prime}\right) \\
& =\sum_{\rho} \operatorname{Re} \frac{1}{s-\rho}-\operatorname{Re} \frac{1}{s-\left(1+i \tau_{0}\right)}-\operatorname{Re} \frac{1}{s-i \tau_{0}} \\
& +O\left(\log \left(Q_{\pi \times \pi^{\prime}}(|s|+2)\right)\right) .
\end{aligned}
$$

Let $s=\sigma+i T$ with $2 \leq \sigma \leq 3$ such that $s \in \mathbb{C}(m)$; this is possible by the structure of $\mathbb{C}(m)$. We want to point out that the left side of $(3.10)$ is $O(1)$. In fact, by RS1, we have for $\operatorname{Re}(s)>1$ that

$$
\frac{d}{d s} \log L\left(s, \pi \times \pi^{\prime}\right)=-\sum_{n=1}^{\infty} \frac{\Lambda(n) a_{\pi}(n) a_{\pi^{\prime}}(n)}{n^{s}} .
$$

From $a_{\pi}\left(p^{k}\right)=\sum_{j=1}^{m} \alpha_{\pi}(j, p)^{k}$ and the bound toward the Ramanujan conjecture proved by Rudnick and Sarnak [17]

$$
\left|\alpha_{\pi}(j, p)\right| \leq p^{1 / 2-1 /\left(m^{2}+1\right)}
$$

we obtain for $n=p^{k}$ that

$$
\left|a_{\pi}(n)\right|=\left|a_{\pi}\left(p^{k}\right)\right| \leq m p^{k\left(1 / 2-1 /\left(m^{2}+1\right)\right)} \leq m n^{1 / 2-1 /\left(m^{2}+1\right)} .
$$

A similar estimate holds for $a_{\pi^{\prime}}(n)$ too. For $n \neq p^{k}$, we have $\Lambda(n)=0$. Thus we conclude that

$$
\sum_{n=1}^{\infty} \frac{\Lambda(n) a_{\pi}(n) \alpha_{\pi^{\prime}}(n)}{n^{s}} \ll_{m} 1
$$

for $\operatorname{Re}(s)=\sigma$ lying between 2 and 3. Therefore from (3.10) we deduce that

$$
\sum_{\rho} \frac{\sigma-\operatorname{Re}(\rho)}{(\sigma-\operatorname{Re}(\rho))^{2}+(T-\operatorname{Im}(\rho))^{2}} \ll \log \left(Q_{\pi \times \pi^{\prime}}(|T|+2)\right) .
$$

This gives the assertion of Lemma 3.1 because $0 \leq \operatorname{Re}(\rho) \leq 1$.

It remains to prove (3.9). We start from the definition of $\Phi\left(s, \pi \times \pi^{\prime}\right)$. By $a_{\pi}(n)=\bar{a}_{\tilde{\pi}}(n)$ and the fact that $\left\{\mu_{\pi \times \pi^{\prime}}(j, k)\right\}=\left\{\bar{\mu}_{\tilde{\pi} \times \tilde{\pi}^{\prime}}(j, k)\right\}$, we have $L\left(s, \tilde{\pi} \times \tilde{\pi}^{\prime}\right)=\bar{L}\left(\bar{s}, \pi \times \pi^{\prime}\right)$ and $L_{\infty}\left(s, \tilde{\pi}_{\infty} \times \tilde{\pi}_{\infty}^{\prime}\right)=\bar{L}_{\infty}\left(\bar{s}, \pi_{\infty} \times \pi_{\infty}^{\prime}\right)$. It follows that

$$
\Phi\left(s, \tilde{\pi} \times \tilde{\pi}^{\prime}\right)=\bar{\Phi}\left(\bar{s}, \pi \times \pi^{\prime}\right),
$$

i.e.,

$$
\begin{aligned}
& \frac{\exp \left(A_{\tilde{\pi} \times \tilde{\pi}^{\prime}}+s B_{\tilde{\pi} \times \tilde{\pi}^{\prime}}\right)}{\left(s-1+i \tau_{0}\right)\left(s+i \tau_{0}\right)} \prod_{\rho_{\tilde{\pi} \times \tilde{\pi}^{\prime}}}\left(1-\frac{s}{\rho}\right) e^{s / \rho} \\
& \quad=\frac{\exp \left(\bar{A}_{\pi \times \pi^{\prime}}+s \bar{B}_{\pi \times \pi^{\prime}}\right)}{\left(s-1+i \tau_{0}\right)\left(s+i \tau_{0}\right)} \prod_{\rho_{\pi \times \pi^{\prime}}}\left(1-\frac{s}{\bar{\rho}}\right) e^{s / \bar{\rho}} .
\end{aligned}
$$


Taking $s=0$ in (3.12), we get

$$
A_{\tilde{\pi} \times \tilde{\pi}^{\prime}}=\bar{A}_{\pi \times \pi^{\prime}}
$$

By $(3.11), \Phi\left(\bar{\rho}, \pi \times \pi^{\prime}\right)=0$ if and only if $\Phi\left(\rho, \tilde{\pi} \times \tilde{\pi}^{\prime}\right)=0$, and consequently

$$
\prod_{\rho_{\pi \times \pi^{\prime}}}\left(1-\frac{s}{\bar{\rho}}\right) e^{s / \bar{\rho}}=\prod_{\rho_{\tilde{\pi} \times \tilde{\pi}^{\prime}}}\left(1-\frac{s}{\rho}\right) e^{s / \rho}
$$

Inserting (3.14) and (3.13) into (3.12), we obtain

$$
B_{\tilde{\pi} \times \tilde{\pi}^{\prime}}=\bar{B}_{\pi \times \pi^{\prime}}
$$

On the other hand, by (3.4) and the functional equation in RS2, we get

$$
\begin{aligned}
B_{\pi \times \pi^{\prime}} & =\frac{d}{d s} \log \Phi\left(0, \pi \times \pi^{\prime}\right)-\frac{1}{1+i \tau_{0}}-\frac{1}{i \tau_{0}} \\
& =-\frac{d}{d s} \log \Phi\left(1, \tilde{\pi} \times \tilde{\pi}^{\prime}\right)+O\left(\log Q_{\pi \times \pi^{\prime}}\right) \\
& =-B_{\tilde{\pi} \times \tilde{\pi}^{\prime}}-\sum_{\rho_{\tilde{\pi} \times \tilde{\pi}^{\prime}}}\left(\frac{1}{1-\rho}+\frac{1}{\rho}\right)+O\left(\log Q_{\pi \times \pi^{\prime}}\right) .
\end{aligned}
$$

Applying (3.15), we conclude

$$
2 \operatorname{Re}\left(B_{\pi \times \pi^{\prime}}\right)=-\sum_{\rho_{\pi \times \pi^{\prime}}}\left(\operatorname{Re} \frac{1}{1-\bar{\rho}}+\operatorname{Re} \frac{1}{\bar{\rho}}\right)+O\left(\log Q_{\pi \times \pi^{\prime}}\right) .
$$

By the functional equation and (3.11), we have $\Phi\left(1-\bar{\rho}, \pi \times \pi^{\prime}\right)=0$ if and only if $\Phi(\rho, \pi \times$ $\left.\pi^{\prime}\right)=0$. Therefore in the above formula we can write $\rho$ in place of $1-\bar{\rho}$, and (3.9) follows.

\section{Estimation of logarithmic derivatives.}

LEMma 4.1. Assume $m=m^{\prime}$ and $\tilde{\pi} \cong \pi^{\prime} \otimes \alpha^{i \tau_{0}}$ for some nonzero $\tau_{0} \in \mathbb{R}$. (a) Let $T>2$. The number $N(T)$ of zeros of $L\left(s, \pi \times \pi^{\prime}\right)$ in the region $0 \leq \operatorname{Re}(s) \leq 1,|\operatorname{Im}(s)| \leq T$ satisfies

$$
N(T+1)-N(T) \ll \log \left(Q_{\pi \times \pi^{\prime}} T\right)
$$

and

$$
N(T) \ll T \log \left(Q_{\pi \times \pi^{\prime}} T\right)
$$

(b) For any $|T|>2$, we have

$$
\sum_{|T-\operatorname{Im}(\rho)|>1} \frac{1}{(T-\operatorname{Im}(\rho))^{2}} \ll \log \left(Q_{\pi \times \pi^{\prime}}|T|\right) .
$$


(c) Let $s=\sigma+i t$ with $-2 \leq \sigma \leq 2,|t|>2$. If $s \in \mathbb{C}(m)$ is not a zero or pole of $L\left(s, \pi \times \pi^{\prime}\right)$, then

$$
\begin{aligned}
& \frac{d}{d s} \log L\left(s, \pi \times \pi^{\prime}\right) \\
& \quad=\sum_{|t-\operatorname{Im}(\rho)| \leq 1} \frac{1}{s-\rho}-\frac{1}{s-1-i \tau_{0}}-\frac{1}{s-i \tau_{0}}+O\left(\log \left(Q_{\pi \times \pi^{\prime}}|t|\right)\right) .
\end{aligned}
$$

(d) For $|T|>2$, there exists $\tau$ with $T \leq \tau \leq T+1$ such that when $-2 \leq \sigma \leq 2$

$$
\frac{d}{d s} \log L\left(\sigma+i \tau, \pi \times \pi^{\prime}\right) \ll \log ^{2}\left(Q_{\pi \times \pi^{\prime}}|\tau|\right) .
$$

(e) For $|T|>2$, there exists $\tau$ with $T \leq \tau \leq T+1$ such that when $-2 \leq \sigma \leq 2$

$$
\frac{d^{2}}{d s^{2}} \log L\left(\sigma+i \tau, \pi \times \pi^{\prime}\right) \ll \log ^{3}\left(Q_{\pi \times \pi^{\prime}}|\tau|\right) .
$$

Proof. (a) The first estimate follows from Lemma 3.1 and the observation that

$$
N(T+1)-N(T)=\sum_{T<\operatorname{Im}(\rho) \leq T+1} 1 \ll \sum_{T<\operatorname{Im}(\rho) \leq T+1} \frac{1}{1+(T-\operatorname{Im}(\rho))^{2}} .
$$

The second inequality can be deduced from the first.

(b) The left side here is less than

$$
2 \sum_{|T-\operatorname{Im}(\rho)|>1} \frac{1}{1+(T-\operatorname{Im}(\rho))^{2}},
$$

which in combination with Lemma 3.1 gives the desired result.

(c) By the structure of $\mathbb{C}(m)$, there exists $2 \leq \sigma_{0} \leq 3$ such that $s_{0}=\sigma_{0}+i t \in \mathbb{C}(m)$. We deduce from (3.7) that

$$
\begin{aligned}
\frac{d}{d s} \log L(s, & \left.\pi \times \pi^{\prime}\right)-\frac{d}{d s} \log L\left(s_{0}, \pi \times \pi^{\prime}\right) \\
= & -\frac{1}{s-1-i \tau_{0}}-\frac{1}{s-i \tau_{0}}+\frac{1}{s_{0}-1-i \tau_{0}}+\frac{1}{s_{0}-i \tau_{0}} \\
& +\sum_{\rho}\left(\frac{1}{s-\rho}-\frac{1}{s_{0}-\rho}\right)+O\left(\log \left(Q_{\pi \times \pi^{\prime}}|t|\right)\right) .
\end{aligned}
$$

Because $\sigma_{0} \geq 2$, the second term on the left side is $O(1)$. By the same reason, $1 /\left(s_{0}-1-\right.$ $\left.i \tau_{0}\right)+1 /\left(s_{0}-i \tau_{0}\right) \ll 1$. By $(\mathrm{a})$, then

$$
\sum_{|\operatorname{Im}(\rho)-t| \leq 1} \frac{1}{s_{0}-\rho} \ll \log \left(Q_{\pi \times \pi^{\prime}}|t|\right) .
$$

Also it follows from (b) and $-2 \leq \sigma \leq 2$ that

$$
\sum_{|\operatorname{Im}(\rho)-t|>1}\left(\frac{1}{s-\rho}-\frac{1}{s_{0}-\rho}\right) \ll \sum_{|\operatorname{Im}(\rho)-t|>1} \frac{1}{(t-\operatorname{Im}(\rho))^{2}} \ll \log \left(Q_{\pi \times \pi^{\prime}}|t|\right) .
$$

Inserting these estimates into (4.1), we get the desired result. 
(d) By the counting of zeros in (a), we can choose $c>0$ and $\tau$ with $T \leq \tau \leq T+1$ satisfying the following properties: For any $\sigma+i t$ with $-2 \leq \sigma \leq 2$ and $|t-\tau| \leq$ $c \log ^{-1}\left(Q_{\pi \times \pi^{\prime}}|T|\right), \sigma+i t \in \mathbb{C}(m), L\left(\sigma+i t, \pi \times \pi^{\prime}\right) \neq 0$, and $\sigma+i t$ is away from poles $1+i \tau_{0}$ and $i \tau_{0}$ of $L\left(s, \pi \times \pi^{\prime}\right)$ by at least $1 / 3$. Then for $s=\sigma+i \tau$

$$
\begin{aligned}
& \frac{d}{d s} \log L\left(s, \pi \times \pi^{\prime}\right) \\
& \ll \frac{1}{c} \log \left(Q_{\pi \times \pi^{\prime}}|\tau|\right) \sum_{|\tau-\operatorname{Im} \rho| \leq 1} 1 \\
& \quad-\frac{1}{s-1-i \tau_{0}}-\frac{1}{s-i \tau_{0}}+O\left(\log \left(Q_{\pi \times \pi^{\prime}}|\tau|\right)\right) \\
& \ll \log ^{2}\left(Q_{\pi \times \pi^{\prime}}|\tau|\right)
\end{aligned}
$$

by (c) and (a).

(e) The proof is similar and easier than that for (d). Now, instead of (3.4), (3.5), and (3.6), we have

$$
\begin{gathered}
\frac{d^{2}}{d s^{2}} \log \Phi\left(s, \pi \times \pi^{\prime}\right)=-\sum_{\rho} \frac{1}{(s-\rho)^{2}}+\frac{1}{\left(s-1-i \tau_{0}\right)^{2}}+\frac{1}{\left(s-i \tau_{0}\right)^{2}}, \\
\frac{d^{2}}{d s^{2}} \log \Phi\left(s, \pi \times \pi^{\prime}\right)=\frac{d^{2}}{d s^{2}} \log L_{\infty}\left(s, \pi_{\infty} \times \pi_{\infty}^{\prime}\right)+\frac{d^{2}}{d s^{2}} \log L\left(s, \pi \times \pi^{\prime}\right),
\end{gathered}
$$

and in $\mathbb{C}(m)$,

$$
\begin{aligned}
& \frac{d^{2}}{d s^{2}} \log L_{\infty}\left(s, \pi_{\infty} \times \pi_{\infty}^{\prime}\right) \\
& \quad=\sum_{j, k} \frac{d^{2}}{d s^{2}} \log \pi^{-\left(s+\mu_{\pi \times \pi^{\prime}}(j, k)\right) / 2}+\sum_{j, k} \frac{d^{2}}{d s^{2}} \log \Gamma\left(\frac{s+\mu_{\pi \times \pi^{\prime}}(j, k)}{2}\right) \\
& \quad \ll \sum_{j, k} \lambda\left(\frac{s+\mu_{\pi \times \pi^{\prime}}(j, k)}{2}\right)^{-1}
\end{aligned}
$$

by (3.3). Then from the definition of $\mathbb{C}(m)$ we get

$$
\frac{d^{2}}{d s^{2}} \log L_{\infty}\left(s, \pi_{\infty} \times \pi_{\infty}^{\prime}\right) \ll_{m} 1
$$

for $s \in \mathbb{C}(m)$. Thus, instead of (3.7), we now have

$$
\begin{aligned}
& \frac{d^{2}}{d s^{2}} \log L\left(s, \pi \times \pi^{\prime}\right) \\
& =\frac{d^{2}}{d s^{2}} \log \Phi\left(s, \pi \times \pi^{\prime}\right)-\frac{d^{2}}{d s^{2}} \log L_{\infty}\left(s, \pi_{\infty} \times \pi_{\infty}^{\prime}\right) \\
& =-\sum_{\rho} \frac{1}{(s-\rho)^{2}}+\frac{1}{\left(s-1-i \tau_{0}\right)^{2}}+\frac{1}{\left(s-i \tau_{0}\right)^{2}}+O(1) .
\end{aligned}
$$


By an argument similar to that leading to (c) we get

$$
\begin{aligned}
& \frac{d^{2}}{d s^{2}} \log L\left(s, \pi \times \pi^{\prime}\right) \\
& \quad=-\sum_{|t-\operatorname{Im}(\rho)| \leq 1} \frac{1}{(s-\rho)^{2}}+\frac{1}{\left(s-1-i \tau_{0}\right)^{2}}+\frac{1}{\left(s-i \tau_{0}\right)^{2}}+O\left(\log \left(Q_{\pi \times \pi^{\prime}}|t|\right)\right)
\end{aligned}
$$

for $s=\sigma+i t$ as in the statement of (c). As in the proof of (d), we can choose $c>0$ and $\tau$ with $T \leq \tau \leq T+1$ satisfying the following properties: For any $\sigma+i t$ with $-2 \leq \sigma \leq 2$ and

$$
|t-\tau| \leq c \log ^{-1}\left(Q_{\pi \times \pi^{\prime}}|T|\right),
$$

$\sigma+i t \in \mathbb{C}(m), L\left(\sigma+i t, \pi \times \pi^{\prime}\right) \neq 0$, and $\sigma+i t$ is away from poles $1+i \tau_{0}$ and $i \tau_{0}$ of $L\left(s, \pi \times \pi^{\prime}\right)$ by at least $1 / 3$. Then the desired result of (e) follows from

$$
\begin{aligned}
\frac{d^{2}}{d s^{2}} \log L & \left(\sigma+i \tau, \pi \times \pi^{\prime}\right) \\
& \ll \frac{1}{c^{2}} \log ^{2}\left(Q_{\pi \times \pi^{\prime}}|\tau|\right) \sum_{|\tau-\operatorname{Im}(\rho)| \leq 1} 1 \\
& \ll \log ^{3}\left(Q_{\pi \times \pi^{\prime}}|\tau|\right)
\end{aligned}
$$

by $(4.2)$.

Lemma 4.2. Assume $m=m^{\prime}$ and $\tilde{\pi} \cong \pi^{\prime} \otimes \alpha^{i \tau_{0}}$ for some nonzero $\tau_{0} \in \mathbb{R}$ as before. If $s$ is in some strip $S_{n}$ as in (3.8) with $n \leq-2$, then

$$
\frac{d^{2}}{d s^{2}} \log L\left(s, \pi \times \pi^{\prime}\right) \ll_{m} 1 .
$$

Proof. By the functional equation in RS2, we have that

$$
\begin{aligned}
& \frac{d^{2}}{d s^{2}} \log L_{\infty}\left(s, \pi_{\infty} \times \pi_{\infty}^{\prime}\right)+\frac{d^{2}}{d s^{2}} \log L\left(s, \pi \times \pi^{\prime}\right) \\
& \quad=\frac{d^{2}}{d s^{2}} \log \varepsilon\left(s, \pi \times \pi^{\prime}\right)+\frac{d^{2}}{d s^{2}} \log L_{\infty}\left(1-s, \tilde{\pi}_{\infty} \times \tilde{\pi}_{\infty}^{\prime}\right) \\
& +\frac{d^{2}}{d s^{2}} \log L\left(1-s, \tilde{\pi} \times \tilde{\pi}^{\prime}\right) .
\end{aligned}
$$

Using the definition of $L_{\infty}\left(s, \pi_{\infty} \times \pi_{\infty}^{\prime}\right)$ and (3.3) we get

$$
\begin{aligned}
\frac{d^{2}}{d s^{2}} \log L_{\infty}\left(s, \pi_{\infty} \times \pi_{\infty}^{\prime}\right) & \\
& =\sum_{j, k} \frac{d^{2}}{d s^{2}} \log \pi^{-\left(s+\mu_{\pi \times \pi^{\prime}}(j, k)\right) / 2}+\sum_{j, k} \frac{d^{2}}{d s^{2}} \log \Gamma\left(\frac{s+\mu_{\pi \times \pi^{\prime}}(j, k)}{2}\right) \\
& \ll \sum_{j, k} \lambda\left(\frac{s+\mu_{\pi \times \pi^{\prime}}(j, k)}{2}\right)^{-1} .
\end{aligned}
$$


As in the proof of Lemma 3.1, for all $s \in \mathbb{C}(m)$ and all $j, k$, we have

$$
\lambda\left(\frac{s+\mu_{\pi \times \pi^{\prime}}(j, k)}{2}\right) \geq \frac{1}{16 m^{2}} .
$$

Thus in $\mathbb{C}(m)$

$$
\frac{d^{2}}{d s^{2}} \log L\left(s, \pi_{\infty} \times \pi_{\infty}^{\prime}\right) \ll_{m} 1
$$

Since $\Phi\left(s, \pi \times \pi^{\prime}\right)=L_{\infty}\left(s, \pi_{\infty} \times \pi_{\infty}^{\prime}\right) L\left(s, \pi \times \pi^{\prime}\right)$ is analytic for Re $s>1$ (RS1) and has only a simple pole $1+i \tau_{0}$ on Re $s=1$ coming from $L\left(s, \pi \times \pi^{\prime}\right)$ (RS3), the Archimedean local factor $L_{\infty}\left(s, \pi_{\infty} \times \pi_{\infty}^{\prime}\right)$ is analytic for $\operatorname{Re} s \geq 1$. Consequently $\operatorname{Re}\left(\mu_{\pi \times \pi^{\prime}}(j, k)\right)>-1$ for all $j, k$. Now we take $s=\sigma+i t \in S_{n}$ with $n \leq-2$ in (4.3); trivially $\sigma<-1$. Thus

$$
\begin{aligned}
\frac{d^{2}}{d s^{2}} \log L & \left(1-s, \tilde{\pi}_{\infty} \times \tilde{\pi}_{\infty}^{\prime}\right) \\
& \ll \sum_{j, k} \lambda\left(\frac{1-s+\mu_{\tilde{\pi} \times \tilde{\pi}^{\prime}}(j, k)}{2}\right)^{-1} \\
& \ll \sum_{j, k} \lambda\left(\frac{1-\sigma+\operatorname{Re}\left(\mu_{\tilde{\pi} \times \tilde{\pi}^{\prime}}(j, k)\right)}{2}\right)^{-1} \\
& \ll_{m} 1 .
\end{aligned}
$$

Using $\sigma<-1$ again, we get

$$
\frac{d^{2}}{d s^{2}} \log L\left(1-s, \tilde{\pi} \times \tilde{\pi}^{\prime}\right) \ll 1 .
$$

The desired result now follows from (4.3)-(4.6).

5. Proof of Theorem 1.2. We will prove Theorem 1.2 when $m=m^{\prime}$ and $\tilde{\pi} \cong$ $\pi^{\prime} \otimes \alpha^{i \tau_{0}}$ for some nonzero $\tau_{0} \in \mathbb{R}$. The case of $\pi$ and $\pi^{\prime}$ being not twisted equivalent was proved by Liu and Ye [12]. By RS1, we have for $\operatorname{Re}(s)>1$ that

$$
\frac{d}{d s} \log L\left(s, \pi \times \tilde{\pi}^{\prime}\right)=-\sum_{n=1}^{\infty} \frac{\Lambda(n) a_{\pi}(n) \bar{a}_{\pi^{\prime}}(n)}{n^{s}},
$$

and therefore

$$
K(s):=\frac{d^{2}}{d s^{2}} \log L\left(s, \pi \times \tilde{\pi}^{\prime}\right)=\sum_{n=1}^{\infty} \frac{(\log n) \Lambda(n) a_{\pi}(n) \bar{a}_{\pi^{\prime}}(n)}{n^{s}} .
$$

By RS3 and RS5, $K(s)$ is holomorphic in $\operatorname{Re}(s)>1$. On $\operatorname{Re}(s)=1, L\left(s, \pi \times \pi^{\prime}\right)$ is nonzero (RS5) and has only a simple pole at $s=1+i \tau_{0}$. Thus

$$
K(s)=\frac{1}{\left(s-1-i \tau_{0}\right)^{2}}+G(s)
$$

has only a double pole in $\operatorname{Re} s \geq 1$, where $G(s)$ is analytic for $\operatorname{Re} s \geq 1$. On $\mathbb{C}, K(s)$ has a double pole at each of the pole at $i \tau_{0}$, trivial zeros, and nontrivial zeros of $L\left(s, \pi \times \pi^{\prime}\right)$. 
Note that

$$
\frac{1}{2 \pi i} \int_{(b)} \frac{y^{s}}{s(s+1)} d s= \begin{cases}1-1 / y & \text { if } y \geq 1 \\ 0 & \text { if } 0<y<1\end{cases}
$$

where $(b)$ means the line $\operatorname{Re}(s)=b>0$. Then we have

$$
\begin{aligned}
\sum_{n \leq x}(1 & \left.-\frac{n}{x}\right) \frac{(\log n) \Lambda(n) a_{\pi}(n) \bar{a}_{\pi^{\prime}}(n)}{n} \\
& =\frac{1}{2 \pi i} \int_{(1)} K(s+1) \frac{x^{s}}{s(s+1)} d s \\
& =\frac{1}{2 \pi i}\left(\int_{1-i T}^{1+i T}+\int_{1-i \infty}^{1-i T} \int_{1+i T}^{1+i \infty}\right) .
\end{aligned}
$$

The last two integrals are clearly bounded by $\ll \int_{T}^{\infty}\left(x / t^{2}\right) d t \ll x / T$. Thus

$$
\begin{aligned}
\sum_{n \leq x} & \left(1-\frac{n}{x}\right) \frac{(\log n) \Lambda(n) a_{\pi}(n) \bar{a}_{\pi^{\prime}}(n)}{n} \\
& =\frac{1}{2 \pi i} \int_{1-i T}^{1+i T} K(s+1) \frac{x^{s}}{s(s+1)} d s+O\left(\frac{x}{T}\right)
\end{aligned}
$$

Choose $\sigma_{0}$ with $-2<\sigma_{0}<-1$ such that the line $\operatorname{Re}(s)=\sigma_{0}$ is contained in the strip $S_{-2} \subset \mathbb{C}(m)$; this is guaranteed by the structure of $\mathbb{C}(m)$. Let $T$ be the $\tau$ such that Lemma 4.1(e) holds. Now we consider the contour

$$
\begin{array}{ll}
C_{1}: & 1 \geq \sigma \geq \sigma_{0}, \quad t=-T \\
C_{2}: & \sigma=\sigma_{0}, \quad-T \leq t \leq T \\
C_{3}: & \sigma_{0} \leq \sigma \leq 1, \quad t=T .
\end{array}
$$

Note that the two poles, some trivial zeros, and certain nontrivial zeros of $L\left(s+1, \pi \times \pi^{\prime}\right)$, as well as $s=0,-1$ are passed by the shifting of the contour. The trivial zeros can be determined by the functional equation in RS2: $s+1=-\mu_{\pi \times \pi^{\prime}}(j, k)$ with $\sigma_{0}<-1-$ $\operatorname{Re}\left(\mu_{\pi \times \pi^{\prime}}(j, k)\right)<0$. Here we used the facts that $\operatorname{Re}\left(\mu_{\pi \times \pi^{\prime}}(j, k)\right)>-1$ and $-2<\sigma_{0}<-1$ 
. Then we have

$$
\begin{aligned}
& \frac{1}{2 \pi i} \int_{1-i T}^{1+i T} K(s+1) \frac{x^{s}}{s(s+1)} d s \\
& =\frac{1}{2 \pi i}\left(\int_{C_{1}}+\int_{C_{2}}+\int_{C_{3}}\right) \\
& +\operatorname{Res}_{s=0,-1} K(s+1) \frac{x^{s}}{s(s+1)} \\
& +\operatorname{Res}_{s=i \tau_{0},-1+i \tau_{0}} K(s+1) \frac{x^{s}}{s(s+1)} \\
& +\sum_{\sigma_{0}<-1-\operatorname{Re}\left(\mu_{\pi \times \pi^{\prime}}(j, k)\right)<0} \operatorname{Res}_{s=-1-\mu_{\pi \times \pi^{\prime}}(j, k)} K(s+1) \frac{x^{s}}{s(s+1)} \\
& +\sum_{|\operatorname{Im}(\rho)| \leq T} \operatorname{Res}_{s=\rho-1} K(s+1) \frac{x^{s}}{s(s+1)} \text {. }
\end{aligned}
$$

By Lemma 4.1(e), we get

$$
\int_{C_{1}} \ll \int_{\sigma_{0}}^{1} \log ^{3}\left(Q_{\pi \times \tilde{\pi}^{\prime}} T\right) \frac{x^{\sigma}}{T^{2}} d \sigma \ll \frac{x \log ^{3}\left(Q_{\pi \times \tilde{\pi}^{\prime}} T\right)}{T^{2}}
$$

and the same upper bound also holds for the integral on $C_{3}$. By Lemma 4.2, then

$$
\int_{C_{2}} \ll \int_{-T}^{T} \frac{x^{\sigma_{0}}}{(|t|+1)^{2}} d t \ll x^{-1}
$$

By taking $T \sim x$, the three integrals in (5.4) are

$$
\ll \frac{\log ^{3}\left(Q_{\pi \times \tilde{\pi}^{\prime}} x\right)}{x}
$$

Obviously, (5.5) is

$$
\operatorname{Res}_{s=0,-1} K(s+1) \frac{x^{s}}{s(s+1)}=K(1)+K(0) x^{-1} .
$$

Since the poles at $s=i \tau_{0}$ and $s=-1+i \tau_{0}$ are double poles, The residues in (5.6) are

$$
\begin{aligned}
\lim _{s \rightarrow i \tau_{0}} & \frac{d}{d s}\left(s-i \tau_{0}\right)^{2} K(s+1) \frac{x^{s}}{s(s+1)} \\
& +\lim _{s \rightarrow-1+i \tau_{0}} \frac{d}{d s}\left(s+1-i \tau_{0}\right)^{2} K(s+1) \frac{x^{s}}{s(s+1)}
\end{aligned}
$$


Using (5.1) we get

$$
\begin{aligned}
\lim _{s \rightarrow i \tau_{0}} & \frac{d}{d s}\left(s-i \tau_{0}\right)^{2} K(s+1) \frac{x^{s}}{s(s+1)} \\
& =\lim _{s \rightarrow i \tau_{0}} \frac{d}{d s}\left(1+\left(s-i \tau_{0}\right)^{2} G(s+1)\right) \frac{x^{s}}{s(s+1)} \\
& =\lim _{s \rightarrow i \tau_{0}} \frac{d}{d s} \frac{x^{s}}{s(s+1)} \ll \log x .
\end{aligned}
$$

Similarly the second term in $(5.11)$ is $\ll(\log x) / x$, and hence $(5.6)$ is bounded

$$
\underset{s=i \tau_{0},-1+i \tau_{0}}{\operatorname{Res}} K(s+1) \frac{x^{s}}{s(s+1)} \ll \log x .
$$

Near a trivial zero $s=-1-\mu_{\pi \times \pi^{\prime}}(j, k)$ of order $l$ in (5.7), we can express $K(s+1)$ as $-l /\left(s+1+\mu_{\pi \times \pi^{\prime}}(j, k)\right)^{2}$ plus an analytic function, like in (5.1). The residues in (5.7) can therefore computed similar to what we did in (5.12). Because $\operatorname{Re}\left(\mu_{\pi \times \pi^{\prime}}(j, k)\right)>-1$ and $-1-\operatorname{Re}\left(\mu_{\pi \times \pi^{\prime}}(j, k)\right)<0,(5.7)$ is bounded

$$
\sum_{\sigma_{0}<-1-\operatorname{Re}\left(\mu_{\pi \times \pi^{\prime}}(j, k)\right)<0} \operatorname{Res}_{s=-1-\mu_{\pi \times \pi^{\prime}}(j, k)} K(s+1) \frac{x^{s}}{s(s+1)} \ll \log x .
$$

To compute the residues corresponding to nontrivial zeros in (5.8), we note that $\Phi(s, \pi \times$ $\left.\tilde{\pi}^{\prime}\right)$ is of order 1 (RS4), and $\Phi\left(1, \pi \times \tilde{\pi}^{\prime}\right) \neq 0$ (RS5). Using an argument like (3.16), we see that

$$
\sum_{\rho} \frac{1}{|\rho(1-\rho)|}<\infty
$$

Consequently, (5.8) becomes

$$
\begin{aligned}
\sum_{|\operatorname{Im}(\rho)| \leq T} & \operatorname{Res}_{s=\rho-1} K(s+1) \frac{x^{s}}{s(s+1)} \\
= & -\sum_{|\operatorname{Im}(\rho)| \leq T} \operatorname{Res}_{s=\rho-1} \frac{1}{(s+1-\rho)^{2}} \frac{x^{s}}{s(s+1)} \\
& \ll \sum_{|\operatorname{Im}(\rho)| \leq T}\left|\frac{x^{\rho-1} \log x}{\rho(\rho-1)}\right| \\
& \ll \log x .
\end{aligned}
$$

Using (5.9), (5.10), and (5.13) through (5.15), we get a bound for (5.3)

$$
\frac{1}{2 \pi i} \int_{1-i T}^{1+i T} K(s+1) \frac{x^{s}}{s(s+1)} d s \ll \log x
$$

The desired estimate (1.9) now follows from this, (5.2), and the fact that $T \sim x$. 


\section{REFERENCES}

[1] J. Arthur and L. Clozel, Simple algebras, base change, and the advanced theory of the trace formula, Annals Math. Studies, 120, Princeton Univ. Press, 1989.

[2] B. Conrey and A. Ghosh, Selberg class of Dirichlet series: Small degrees, Duke Math. J., 72 (1993), 673-693.

[3] H. Davenport, Multiplicative Number Theory, 2nd ed. Springer, Berlin 1980.

[4] S. Gelbart and F. Shahidi, Boundedness of automorphic L-functions in vertical strips, J. Amer. Math. Soc., 14 (2001), 79-107.

[5] R. Godement and H. Jacquet, Zeta functions of simple algebras, Lecture Notes in Math., 260, Springer-Verlag, Berlin, 1972.

[6] H. Jacquet, I.I. Piatetski-Shapiro, and J. Shalika, Rankin-Selberg convolutions, Amer. J. Math., 105 (1983), 367-464.

[7] H. Jacquet and J.A. Shalika, On Euler products and the classification of automorphic representations I, Amer. J. Math., 103 (1981), 499-558.

[8] H. Jacquet and J.A. Shalika, On Euler products and the classification of automorphic representations II, Amer. J. Math., 103 (1981), 777-815.

[9] H. Kim, Functoriality for the exterior square of $G L_{4}$ and the symmetric fourth of $G L_{2}$, J. Amer. Math. Soc., 16 (2003), 139-183.

[10] H. Kim and P. Sarnak, Refined estimates towards the Ramanujan and Selberg conjectures, Appendix to Kim [9].

[11] R.P. Langlands, Problems in the theory of automorphic forms, in: Lectures in Modern Analysis and Applications III, Lect. Notes Math., 170 (1970), 18-61.

[12] Jianya Liu and Yangbo Ye, Superposition of zeros of distinct L-functions, Forum Math., 14 (2002), 419-455.

[13] C. Moeglin and J.-L. Waldspurger, Le spectre résiduel de $G L(n)$, Ann. Sci. École Norm. Sup., (4) 22 (1989), 605-674.

[14] M. Ram Murty, Selberg's conjectures and Artin L-functions , Bull. Amer. Math. Soc., 31 (1994), 1-14.

[15] M. Ram Murty, Selberg's conjectures and Artin L-functions II, Current trends in mathematics and physics, Narosa, New Delhi, 1995, 154-168.

[16] C.D. Pan and C.B. Pan, Fundamentals of analytic number theory, Science Press, 1991.

[17] Z. Rudnick and P. Sarnak, Zeros of principal L-functions and random matrix theory, Duke Math. J., 81 (1996), 269-322. 
[18] A. Selberg, Old and new conjectures and results about a class of Dirichlet series, Collected Papers, vol. II, Springer, 1991, 47-63.

[19] F. Shahidi, On certain L-functions, Amer. J. Math., 103 (1981), 297-355.

[20] F. Shahidi, Fourier transforms of intertwining operators and Plancherel measures for GL(n), Amer. J. Math., 106 (1984), 67-111.

[21] F. Shahidi, Local coefficients as Artin factors for real groups, Duke Math. J., 52 (1985), 973-1007.

[22] F. Shahidi, A proof of Langlands' conjecture on Plancherel measures; Complementary series for p-adic groups, Ann. Math., 132 (1990), 273-330.

Jianya Liu: Department of Mathematics, Shandong University

JiNAN 250100, ChINA. jyliu@sdu.edu.cn

Yangbo Ye: Department of Mathematics, The University of Iowa

Iowa City, Iowa 52242-1419, USA. yey@math.uiowa.edu 